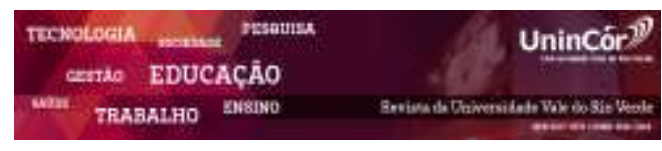

Revista da Universidade Vale do Rio Verde ISSN: 1517-0276 / EISSN: 2236-5362 Vol. 16 | n. 1 | Ano 2018

Amanda Campos Damasceno Faculdade de Medicina, UNIFENAS/Alfenas amandacdamasceno2009@hotmail.com

Cláudio Daniel Cerdeira Instituto de Ciências Biomédicas, Universidade Federal de Alfenas (UNIFAL-MG) daniel.cerdeira.84@gmail.com

Bruna Loss de Souza Faculdade de Medicina, UNIFENAS/Alfenas brunaloss@live.com

Ingryd Pereira de Andrade Faculdade de Medicina, UNIFENAS/Alfenas ingrydinhabloom@outlook.com

Julielly Almeida Andrade Brandão Faculdade de Medicina, UNIFENAS/Alfenas juliely.almeida@hotmail.com

Karen Ávila Souza Faculdade de Medicina, UNIFENAS/Alfenas karenavilasouza@gmail.com

Marina Silveira Duarte Faculdade de Medicina, UNIFENAS/Alfenas nina_zd2010@hotmail.com

Samyra Nina Serra

Faculdade de Medicina, UNIFENAS/Alfenas samyra.net@gmail.com

Gérsika Bitencourt Santos Barros Faculdade de Medicina, UNIFENAS/Alfenas gersikasantos@unifenas.br

\section{DISPENSAÇÃO DE INSULINA EM UMA \\ UNIDADE DO SISTEMA ÚNICO DE SAÚDE EM UM MUNICÍPIO \\ BRASILEIRO NO SUL DE MINAS GERAIS}

\section{RESUMO}

Diabetes mellitus (DM) é um grupo heterogêneo de distúrbios endócrino-metabólicos que tem como característica comum o acúmulo de glicose pelo organismo. Este é um estudo observacional, descritivo/quantitativo e transversal no qual foi avaliado a dispensação de diferentes tipos de insulina, entre Janeiro e Dezembro de 2015, em um Centro de Distribuição de Medicamentos (CDM) do Sistema Único de Saúde (SUS), em Alfenas, Minas Gerais, Brasil. As insulinas foram dispensadas para pacientes com diferentes tipos de DM (DM tipo 1, DM tipo 2, outros tipos específicos de DM ou DM gestacional), tendo ocorrido uma maior dispensação para pacientes do sexo feminino. A insulina mais dispensada foi a NPH (3948 dispensações, 65,6\% do total), devido a sua ação intermediária, seguida das associações (misturas): NPH e Lispro (801 dispensações, 13,3\%); NPH e Regular (580 dispensações, 9,6\%), sendo que as insulinas Lispro e Regular possuem ação rápida e curta, respectivamente, e estas misturas são utilizadas antes das refeições. Houve 332 dispensações de Lispro e 321 de Glargina (uma insulina de ação longa). A insulina NPH foi mais utilizada por pacientes da faixa etária acima dos 61 anos (2057 dispensações, 34,2\% do total), devido principalmente ao seu custo benefício e posologia. Portanto, atualmente é elevado o número de pacientes que utilizam diversos tipos de insulinas (inclusive as associações) disponibilizadas por uma CDM. Estas dispensações levam em conta os fatores farmacocinéticos de cada insulina, com propósitos de evitar picos de hipoglicemia durante o dia, facilitar a aderência do paciente ao tratamento e consequentemente aumentar a efetividade terapêutica, com a utilização da insulina que seja mais adequada aos hábitos cotidianos e perfil de DM de cada paciente.

Palavras-chave: Insulina. NPH. Dispensação. Diabetes mellitus.

\begin{abstract}
Diabetes mellitus (DM) is a group of endocrine and metabolic diseases markedly characterized by hyperglycemia. In this observational, descriptive/quantitative, and cross-sectional study, the dispensing of different types of insulin at a drug distribution center of the Unified Health System was evaluated, between
\end{abstract}


January and December, 2015, in Alfenas city, Minas Gerais, Brazil. The insulins were dispensed to patients with different types of DM (type $1 \mathrm{DM}$, type $2 \mathrm{DM}$, other specific types of DM, or gestational DM), and most of the dispensations to patients from the feminine sex. The most dispensed insulin was the NPH (3948 dispensations, $65.6 \%$ from the total), because its intermediateacting, it follows by the insulin mixtures: NPH and Lispro (801 dispensations, 13.3\%); NPH and Regular (580 dispensations, $9.6 \%$ ), being that these alone NPH-associated insulins present rapid-acting and short-acting, respectively, and they are injected before the meals. The Lispro alone and Glargine alone (longacting) accounted for 332 and 321 dispensations, respectively. The insulin NPH was the most used by the patients older than 61 years (2057 dispensations, $34.2 \%$ from the total), chiefly due to the cost-effectiveness and posology. Therefore, currently is greater the number of patients using different types of insulin (including the mixtures) that are dispensed at a drug distribution center. The dispensing take into account pharmacokinetic factors from the insulins, avoiding hypoglycemia peaks during all day and easing patient adherence to the treatment accordingly increasing therapeutic efficacy, with utilization of the insulin most suitable to daily habits and DM profile of each patient.

Keywords: Insulin. NPH. Dispensing. Diabetes mellitus.

Recebido em: 02/10/2017 - Aprovado em: 10/01/2018- Disponibilizado em: 15/07/2018

\section{INTRODUÇÃO}

Diabetes mellitus (DM) é um grupo heterogêneo de distúrbios endócrino-metabólicos que apresenta em comum à hiperglicemia, tendo sua gênese nos defeitos na secreção de insulina pelo pâncreas (DM tipo 1), defeitos na ação da insulina em seus alvos celulares (DM tipo 2), ou ambos os eventos. O DM tipo 1, também conhecido como diabete autoimune ou juvenil, normalmente aparece na infância ou adolescência, sendo necessário o uso de insulina diariamente. No DM tipo 2 o organismo passa, ao longo do tempo, a ter deficiência na secreção da insulina, devido a uma dieta irregular (DIRETRIZES DA SOCIEDADE BRASILEIRA DE DIABETES, 2016; SILVA et al., 2016).
Atualmente, especialistas alertam que uma epidemia de DM ainda está em curso. Estima-se que a população mundial com diabetes seja da ordem de 387 milhões e que alcance 471 milhões em 2035, em virtude do crescimento e do envelhecimento populacional, da maior urbanização, da progressiva prevalência de obesidade e sedentarismo, bem como da maior sobrevida de pacientes com DM. Ainda, cerca de $80 \%$ dos afetados vivem em países em desenvolvimento, onde a epidemia tem maior intensidade e há uma crescente proporção de pessoas acometidas em grupos etários mais jovens (DIRETRIZES DA SOCIEDADE BRASILEIRA DE DIABETES, 2016). No Brasil, cerca de 8\% da população possuem DM tipo 1 e, o DM tipo 2 tem a maior incidência no país, sendo que $33 \%$ da população entre 60 e 79 anos possuem algum tipo 
de DM ou alguma alteração relacionada ao metabolismo da glicose (DIRETRIZES DA SOCIEDADE BRASILEIRA DE DIABETES, 2016).

A classificação atual do DM é baseada na etiologia e não no tipo de tratamento, portanto, os termos "DM insulino-dependente" e "DM insulino-independente" devem ser evitados. Assim, a classificação proposta pela Organização Mundial da Saúde (OMS) e pela Associação Americana de Diabetes (ADA), inclui quatro classes clínicas: DM tipo 1, DM tipo 2, outros tipos específicos de DM e DM gestacional. Existem também outras duas categorias, referidas como pré-diabetes, que são a glicemia de jejum alterada e a tolerância à glicose diminuída (DIRETRIZES DA SOCIEDADE BRASILEIRA DE DIABETES, 2016). Assim, baseado no acima exposto, condições de saúde e hábitos de vida do paciente, a terapêutica do DM é direcionada.

Em 1922, pesquisadores canadenses foram os primeiros a demonstrar os efeitos benéficos da insulina em corrigir o metabolismo da glicose no DM tipo 1 e, em 1936, após a descoberta que a adição da proteína protamina e zinco prolongam a ação da insulina convencional, o conceito de insulina intermediária (insulina NPH, do inglês neutral protamine Hagedorn) foi posteriormente estabelecido, na década de 1950 (BINDER \& BRANGE, 1997; DONNER, 2015).

Em 1955, a estrutura da insulina foi totalmente sequenciada, evidenciado a presença de 51 aminoácidos, 21 dispostos em uma cadeia A e 30 em uma B, sendo estas unidas por duas pontes dissufeto (entre A7 e B7 e entre A20 e B19), cuja natureza proteica explica a impossibilidade de administrado deste medicamento por via oral. Na década de 1980, com o uso da tecnologia do DNA recombinante e a consequente introdução das insulinas humanas no mercado (a principio, obtida através da inserção do gene da pró-insulina humana em Saccharomyces cerevisiae ou Escherichia coli não patogênica), o uso de insulinas de origens suína ou bovina declinaram (BINDER \& BRANGE, 1997; HORVATH et al., 2007; DONNER, 2015).

Na década de 1990, foi possível introduzir no mercado insulinas humanas análogas, visando alterações farmacocinéticas que propiciassem uma melhor eficácia durante a terapêutica do DM. Apoiada em prévios estudos de relação estrutura atividade (REA), que mostraram que as regiões B26-B30 não são criticas para o reconhecimento e interação com os receptores celulares da insulina, foi possível utilizar a tecnologia do DNA recombinante para promover inserções de aminoácidos e/ou outras moléculas e/ou fazer alterações nestas regiões (BINDER \& BRANGE, 1997; DONNER, 2015).

Assim, foram obtidas as insulinas Lispro (inserção de Lisina em B28 e Prolina em B29) e Glargina (inserção de duas Argininas em B30 e substituição de Aspargina por Glicina em A20). Estes análogos são classificados quanto as suas características farmacocinéticas, sendo estas: ações rápida (Lispro), curta (Regular humana), intermediária (NPH) e longa (Glargina). Como uma consequência, NPH é a insulina padrão na terapia do DM tipo 2. Além disso, foram possíveis as criações de misturas destas insulinas, podendo ser encontradas no mercado as associações que combinam características farmacocinéticas ideais, incluindo NPH/Regular, NPH/Regular, 
Lispro/Glargina e NPH/Lispro/Regular (BINDER \& BRANGE, 1997; HORVATH et al., 2007; DONNER, 2015).

O tratamento do DM envolve mudanças no estilo de vida e pode exigir uma intervenção farmacológica com insulina ou agentes hipoglicemiantes orais (YOUNIS et al., 2001; SILVA et al., 2016), ocorrendo no mercado, como mencionado, uma variedade de preparações altamente purificadas para tratar esta condição crônica, que devem ser usadas diariamente por um longo período de tempo ou até mesmo por toda a vida do paciente (GOLDMAN \& AUSIELLO, 2014).

Neste contexto, em 2004, o Governo Federal criou o Programa "Farmácia Popular do Brasil" no âmbito do Sistema Único de Saúde (SUS), com o objetivo de ampliar o acesso da população aos medicamentos essenciais. Este é um sistema voltado à atenção à saúde e educação quanto ao uso racional dos medicamentos e destinado aos pacientes que tem dificuldade em adquirir os medicamentos que necessitam, sendo necessário que os usuários apresentem uma receita prescrita por um médico da rede privada ou SUS (FURIERI, 2009).

Atualmente é elevado o número de pacientes que utilizam insulina como tratamento antidiabético através do programa farmácia popular. Baseado nas considerações acima, o presente estudo avaliou a dispensação de quatro tipos de insulinas (NPH, Lispro, Regular e Glargina) e suas associações, em um Centro de Distribuição de Medicamentos (CDM) do SUS em Alfenas, no sul de Minas Gerais, Brasil, com expectativa que esta proposta possa identificar a frequência e os fatores associados ao uso de insulina pelos pacientes atendidos.

\section{MÉTODOS}

Este estudo foi realizado de acordo com os padrões éticos constantes na Declaração de Helsinki e, suas atualizações, e as Normas de Pesquisa Envolvendo Seres Humanos do Conselho Nacional de Saúde (CNS) do Brasil (Res. CNS 196/96), sendo previamente aprovado pelo comitê local de ética em pesquisa envolvendo humanos (Protocolo de aprovação: CAAE 44329715.3.0000.5143, parecer $n^{\circ}$ 1.092.525). O protocolo do estudo envolvendo humanos foi desenhado para proteger os envolvidos neste estudo.

Este é um estudo transversal e observacional. Com o intuito de identificar as insulinas mais dispensadas para a população da cidade de Alfenas, durante o período de Janeiro a Dezembro de 2015, foram utilizados dados do relatório de dispensação de insulinas fornecidos pelo CDM dessa cidade. Baseando-se na população total do município (segundo o IBGE [2013], possui uma população total de 73.774 habitantes), na prevalência estimada de pacientes diabéticos atendidos pelo SUS de Alfenas, foi realizada uma amostragem estratificada para calculo do tamanho da amostra, considerando um intervalo de confiança de 95\%. Foram incluídos no estudo: Pacientes com todos os tipos de DM (DM tipo 1, DM tipo 2, outros tipos específicos de DM ou DM gestacional) atendidos no período supracitado. Os dados coletados foram: relação quanto ao sexo e idade dos portadores de DM, tipo de insulina e as quantidades dispensadas durante todos os meses de 2015, a cada paciente. Os dados foram analisados e apresentados como gráficos e/ou tabelas. 


\section{RESULTADOS E DISCUSSÃO}

O presente estudo avaliou a dispensação de insulinas utilizadas por pacientes de ambos os sexos com diferentes tipos de DM (DM tipo 1, DM tipo 2, outros tipos específicos de DM ou DM gestacional), atendidos em uma CDM do SUS, em Alfenas-MG, Brasil. Foram observadas 6019 dispensações entre Janeiro a Dezembro de 2015. Quanto ao sexo, para as mulheres, a dispensação entre janeiro a maio variou entre 61 a $63 \%$, sendo que em junho caiu para 37\%, já em julho foi $61 \%$, agosto $62 \%$, setembro $59 \%$, outubro $39 \%$, novembro voltou para $63 \%$ e em dezembro teve seu maior índice, 65\%. Para os homens foi verificado menor dispensação na maioria dos meses, sendo que entre janeiro a maio variou entre 38 e 39\%, enquanto em junho houve um considerável aumento, para $63 \%$, em julho voltou para $39 \%$, agosto $38 \%$, setembro $41 \%$, outubro subiu novamente para $61 \%$, novembro voltou para $37 \%$ e em dezembro teve seu índice mais baixo, com $37 \%$ de dispensação. Estes dados são apresentados na Tabela 1, onde em geral, pode ser observado que as mulheres utilizaram uma maior quantidade de insulina do que os homens, o que pode indicar o maior cuidado das mulheres, que fazem o uso contínuo de insulina, visando uma melhor qualidade de vida, também evidenciado pelo fato que mulheres tendem a buscar mais os serviços de saúde preventivos.

A Figura 1 apresenta o número e tipos de insulinas dispensadas na CDM avaliada, demonstrando que os tipos de insulina foram: NPH, Lispro, Glargina, Regular, NPH e Lispro, NPH e Regular, Lispro e Glargina, NPH, Lispro e Regular. Como observado, a NPH foi a mais dispensada.

Complementarmente, como mostrado na

Tabela 2, foi avaliada a relação entre os tipos de insulinas dispensadas no ano de 2015 e a faixa etária dos pacientes usuários destes medicamentos, onde é observado que entre as idades de 0 a 20 anos, o tipo de insulina mais dispensada foi a Glargina, entre 21 a 40 anos a NPH e entre 41 a 60 anos e acima de 61 anos a NPH. A NPH foi à insulina mais dispensada, provavelmente por conta do seu período de duração ser intermediário e o seu custo financeiro ser considerado baixo (BINDER \& BRANGE, 1997; HORVATH et al., 2007; DONNER, 2015). 
Tabela 1 - Relação (\%) de insulinas dispensadas de acordo com o sexo dos pacientes, entre Janeiro e Dezembro de 2015 na CDM de Alfenas, MG, Brasil.

\begin{tabular}{|c|c|c|c|c|c|c|}
\hline \multirow[t]{2}{*}{ Sexo } & \multicolumn{6}{|c|}{ Meses (2015) } \\
\hline & Janeiro & Fevereiro & Março & Abril & Maio & Junho \\
\hline Feminino & $61 \%$ & $63 \%$ & $62 \%$ & $61 \%$ & $62 \%$ & $37 \%$ \\
\hline Masculino & $39 \%$ & $37 \%$ & $38 \%$ & $39 \%$ & $38 \%$ & $63 \%$ \\
\hline \multirow[t]{2}{*}{ Sexo } & \multicolumn{6}{|c|}{ Meses (2015) } \\
\hline & Julho & Agosto & Setembro & Outubro & Novembro & Dezembro \\
\hline Feminino & $61 \%$ & $62 \%$ & $59 \%$ & $39 \%$ & $63 \%$ & $65 \%$ \\
\hline Masculino & $39 \%$ & $38 \%$ & $41 \%$ & $61 \%$ & $37 \%$ & $35 \%$ \\
\hline
\end{tabular}

Fonte: Prontuários da CDM de Alfenas, 2015.

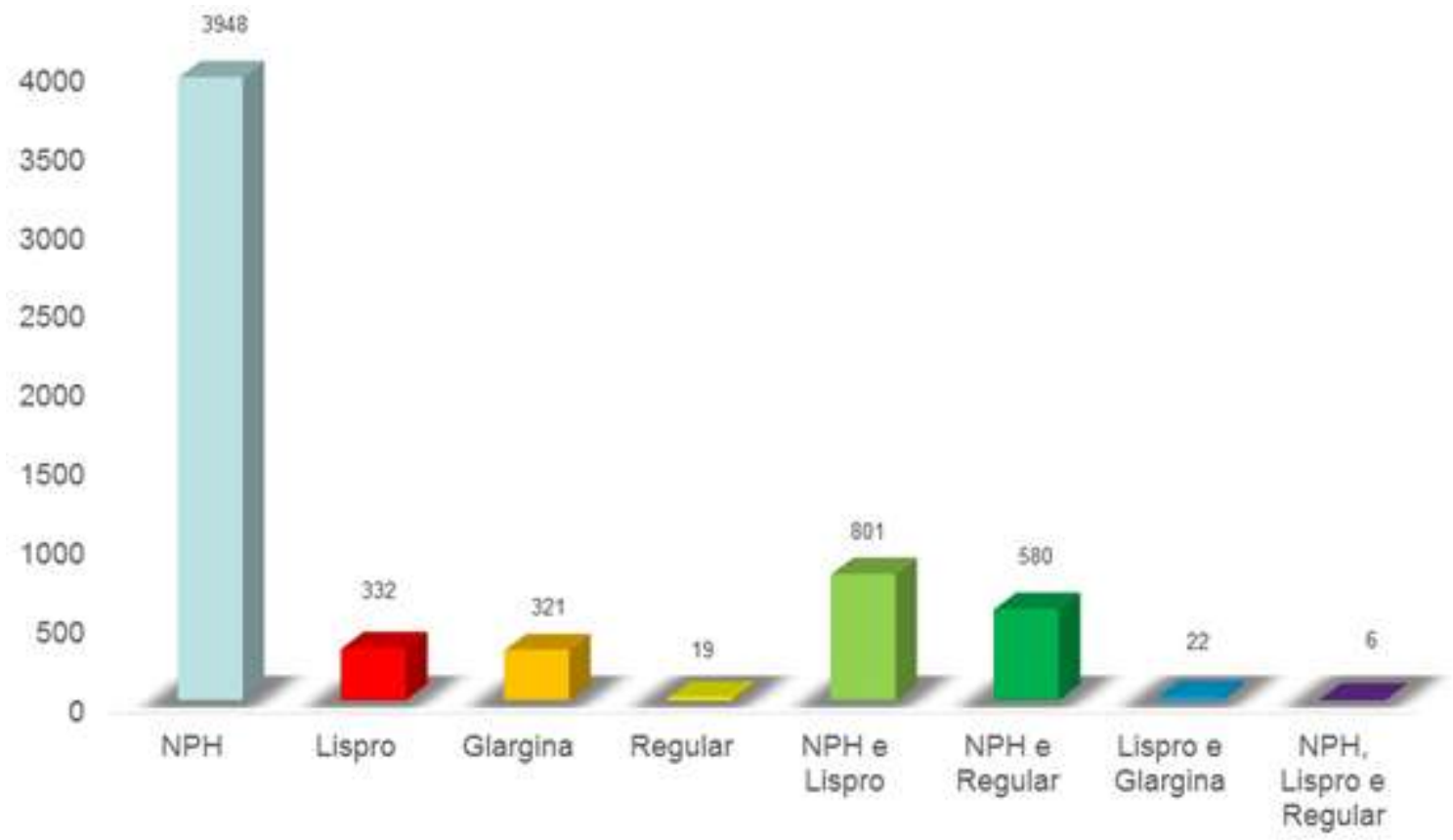

Figura 1 - Número total (valores absolutos, vertical) e tipos (horizontal) de insulinas dispensadas entre Janeiro e Dezembro de 2015 na CDM de Alfenas, MG, Brasil.

Fonte: Prontuários da CDM de Alfenas, 2015. 
Tabela 2 - Relação entre os tipos de insulinas (valores absolutos) dispensadas no ano de 2015 e a faixa etária dos pacientes usuários.

\begin{tabular}{|c|c|c|c|c|}
\hline \multirow{2}{*}{$\begin{array}{c}\text { Insulinas } \\
\text { dispensadas } 2015\end{array}$} & \multicolumn{4}{|c|}{ Faixas etárias } \\
\hline & $0-20$ anos & 21 a 40 anos & 41 a 60 anos & Mais de 61 anos \\
\hline NPH & 88 & 284 & 1519 & 2057 \\
\hline Lispro & 89 & 101 & 66 & 76 \\
\hline Glargina & 129 & 84 & 58 & 50 \\
\hline Regular & 0 & 0 & 7 & 12 \\
\hline NPH e Lispro & 79 & 226 & 248 & 248 \\
\hline NPH e Regular & 28 & 56 & 225 & 271 \\
\hline Lispro e Glargina & 9 & 5 & 5 & 3 \\
\hline $\begin{array}{l}\text { NPH, Lispro e } \\
\text { Regular }\end{array}$ & 0 & 2 & 0 & 4 \\
\hline
\end{tabular}

Fonte: Prontuários da CDM de Alfenas, 2015.

A incidência de DM no Brasil é alta, e varia consideravelmente de acordo de acordo com a faixa etária avaliada (MALERBI \& FRANCO, 1992). Devido às condições sócio-econômicas de grande parte da população afetada pelo DM, os CDMs têm propiciado tratamento adequado a grande parcela da população, que estão sob o uso diário de medicamentos para controlar esta doença crônica. Ainda, o tratamento deve ser especifico as condições do paciente bem como a sua rotina, facilitando a adesão e aumentando a efetividade terapêutica. Como terapia única, neste estudo, nós observamos que a insulina NPH foi majoritariamente a mais dispensada (3948 dispensações), seguida da Lispro (332 dispensações), Glargina (321 dispensações) e Regular (19 dispensações).

A insulina Lispro é considerada rápida, com início de ação entre 15 a 30 minutos, e duração curta, de 2 a 3 horas. A insulina regular é considerada de ação curta, com início do efeito entre 30 minutos à uma hora, porém a duração também é curta, que varia de 3 a 6 horas. A insulina NPH é uma insulina de ação intermediária, que tem início de ação de 1 a 3 horas e uma maior duração, de 12 a 24 horas. A insulina glargina corresponde a uma insulina de ação longa (24 horas), o que corresponderia à insulina basal (FREITAS et al., 2006). Estas insulinas são prescritas de acordo com as características do paciente, visando facilitar a aderência e efetividade terapêutica (BINDER \& BRANGE, 1997; HORVATH et al., 2007).

Como visto neste estudo, as misturas de insulinas durante a terapêutica do DM contam por grande parte das dispensações, tendo sido observado para NPH e Lispro (801 dispensações), NPH e Regular (580 dispensações), Lispro e Glargina (22 dispensações) e NPH, Lispro e Regular (6 dispensações). Normalmente, são encontradas misturas entre insulinas consideradas 
rápidas ou curtas com insulinas de ação intermediária ou longa. Essas combinações têm por objetivo mimetizar a secreção fisiológica de insulina (BINDER \& BRANGE, 1997; HORVATH et al., 2007; DONNER, 2015). As combinações de insulina NPH e regular falham nesta mimetização pelo fato da insulina regular necessitar de 30 minutos à uma hora para fazer efeito, sendo utilizada de meia à uma hora antes das refeições, porém sua ação continua por até seis horas, podendo ocasionar, juntamente com a insulina de ação intermediária, picos hipoglicêmicos nos pacientes. A combinação de lispro e NPH tem o mesmo objetivo que a combinação entre NPH e regular. A utilização da insulina glargina no período noturno como insulina basal faz com que os níveis de glicemia em jejum sejam menores, comparando-se com outras insulinas basais, o que gera menores chances de pico hipoglicêmico.

\section{CONCLUSÃO}

Neste estudo, nós observamos que atualmente é elevado o número de pacientes que utilizam diversos tipos de insulinas dispensadas por um CDM (inclusive as combinações), considerando a duração de cada uma, com os propósitos de evitar picos de hipoglicemia durante o dia, facilitar a aderência do paciente ao tratamento e, consequentemente, aumentar a efetividade terapêutica. Este estudo também demonstrou que a insulina mais dispensada foi a NPH, seguida das associações NPH e Lispro e NPH e Regular. A maior dispensação de NPH foi provavelmente por conta de sua ação intermediária. Além disso, este medicamento é disponibilizado a um baixo custo, o que aumenta a preferência de prescrição. As associações, que contaram por grande parte das dispensações, foram utilizadas por agirem tanto no dia todo quanto antes das refeições. É importante ressaltar que para que o paciente se torne aderente ao tratamento é necessário que ele utilize a insulina que seja mais adequada ao seu cotidiano, aos seus hábitos, bem como seu perfil de DM.

\section{REFERÊNCIAS}

1. DIRETRIZES DA SOCIEDADE BRASILEIRA DE DIABETES (2015-2016)/Adolfo Milech...[et al.]; organização José Egidio Paulo de Oliveira, Sérgio Vencio - São Paulo: A.C. Farmacêutica; 2016.

2. SILVA, A. R.; CERDEIRA, C. D.; BRITO, A. R.; SALLES, B. C. C.; RAVAZI, G. F.; MORAES, G. O. I.; RUFINO, L. R. A.; OLIVEIRA, R. B. S.; SANTOS, G. B. Green banana pasta diet prevents oxidative damage in liver and kidney and improves biochemical parameters in type 1 diabetic rats. Arch Endocrinol Metab, v. 60, n. 4, p. 355-366, 2016.

3. HORVATH, K.; JEITLER, K.; BERGHOLD, A.; EBRAHIM, S. H.; GRATZER, T. W.; PLANK, J.; KAISER, T.; PIEBER, T. R.; SIEBENHOFER, A. Long-acting insulin analogues versus NPH insulin (human isophane insulin) for type 2 diabetes mellitus. Cochrae Database of Systematic Reviews, v. 2(CD005613), 2007.

4. BINDER, C.; BRANGE, J. Insulin chemistry and pharmacokinetics. In: PORTE, D. J. R.; SHERWIN, $\mathrm{R}$ (eds)

ELLENBERG'S AND RIFKIN'S Diabetes Mellitus, 5th edition ed. Appleton and Lange, Stam ford, CT, p 689; 1997.

5. DONNER, C. T. Insulin-Pharmacology, Therapeutic Regimens and Principles of Intensive Insulin Therapy. Endotext- NCBI Bookshelf. (Last Update); 2015.

6. YOUNIS, W. S.; CAMPBELL, S.; SLACK, M. K. Pharmacists' Attitudes Toward Diabetes and Their Involvement in Diabetes Education. Ann Pharmacother, v. 35, n. 7, p. 841-845, 2001. 
7. GOLDMAN, L.; AUSIELLO, D. C: Tratado de Medicina Interna. $24^{\text {a }}$ Edição. Rio de Janeiro: Elsevier; 2014.

8. FURIERI, L. V. Dispensação Farmacêutica: um modelo aplicado na Farmácia Popular do Brasil. 2009. 38 f. (Conclusão de Curso) - Faculdade de Ciências Biológicas, Centro Universitário Newton Paiva, Belo Horizonte, 2009.

9. MINISTÉRIO DA SAÚDE (Brasil). Secretaria de Vigilância em Saúde. Departamento de Análise de Situação de Saúde. A vigilância, o controle e a prevenção das doenças crônicas não transmissíveis. DCNT no contexto do Sistema Único de saúde brasileiro - Situação e desafios atuais. Brasília: OPAS, 80 p; 2005a.

10. MALERBI, D. A.; FRANCO, L. J. Multicenter study of the prevalence of diabetes mellitus and impaired glucose tolerance in the urban Brazilian population aged 30-69 Yr. Diabetes Care, v. 15, p. 1509-1516, 1992.

\section{FREITAS, E. V.; et al. Tratado de Geriatria e} Gerontologia. 2 ed. Rio de Janeiro: Guanabara Koogan; 2006.

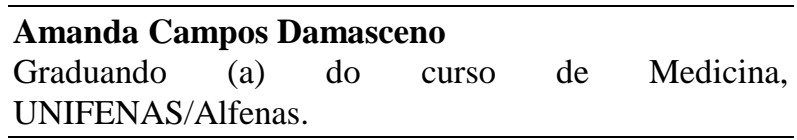

\section{Cláudio Daniel Cerdeira}

Doutorando em Ciências Farmacêuticas, Universidade Federal de Alfenas (UNIFAL-MG).

\section{Bruna Loss de Souza}

Graduanda do curso de Medicina, UNIFENAS/Alfenas.

\section{Ingryd Pereira de Andrade}

Graduanda do curso de Medicina, UNIFENAS/Alfenas.

\section{Julielly Almeida Andrade Brandão}

Graduanda do curso de Medicina, UNIFENAS/Alfenas.

\section{Karen Ávila Souza}

Graduanda do curso de Medicina, UNIFENAS/Alfenas.
Samyra Nina Serra

Graduanda do curso de Medicina, UNIFENAS/Alfenas.

Gérsika Bitencourt Santos Barros

Professora do curso de Medicina, UNIFENAS/Alfenas.

\section{Marina Silveira Duarte}

Graduanda do curso de Medicina, UNIFENAS/Alfenas. 